\title{
Image Fusion using Complex Wavelets
}

\author{
Paul Hill, Nishan Canagarajah and Dave Bull \\ Dept. of Electrical and Electronic Engineering \\ The University of Bristol \\ Bristol, BS5 1UB, UK \\ paul.hill, dave.bull, nishan.canagarajah@bristol.ac.uk
}

\begin{abstract}
The fusion of images is the process of combining two or more images into a single image retaining important features from each. Fusion is an important technique within many disparate fields such as remote sensing, robotics and medical applications. Wavelet based fusion techniques have been reasonably effective in combining perceptually important image features. Shift invariance of the wavelet transform is important in ensuring robust subband fusion. Therefore, the novel application of the shift invariant and directionally selective Dual Tree Complex Wavelet Transform (DT-CWT) to image fusion is now introduced. This novel technique provides improved qualitative and quantitative results compared to previous wavelet fusion methods.
\end{abstract}

\section{Introduction}

Image fusion is the process by which two or more images are combined into a single image retaining the important features from each of the original images. The fusion of images is often required for images acquired from different instrument modalities or capture techniques of the same scene or objects. Important applications of the fusion of images include medical imaging, microscopic imaging, remote sensing, computer vision, and robotics.

Fusion techniques include the simplest method of pixel averaging to more complicated methods such as principal component analysis and wavelet transform fusion. Several approaches to image fusion can be distinguished, depending on whether the images are fused in the spatial domain or they are transformed into another domain, and their transforms fused. 


\section{Wavelet Transform Fusion}

The most common form of transform image fusion is wavelet transform fusion $[1,2,4$, $6,9]$. In common with all transform domain fusion techniques the transformed images are combined in the transform domain using a defined fusion rule then transformed back to the spatial domain to give the resulting fused image. Wavelet transform fusion is more formally defined by considering the wavelet transforms $\omega$ of the two registered input images $I_{1}(x, y)$ and $I_{2}(x, y)$ together with the fusion rule $\phi$. Then, the inverse wavelet transform $\omega^{-1}$ is computed, and the fused image $I(x, y)$ is reconstructed:

$$
I(x, y)=\omega^{-1}\left(\phi\left(\omega\left(I_{1}(x, y)\right), \omega\left(I_{2}(x, y)\right)\right)\right) .
$$

This process is depicted in figure $1^{1}$.

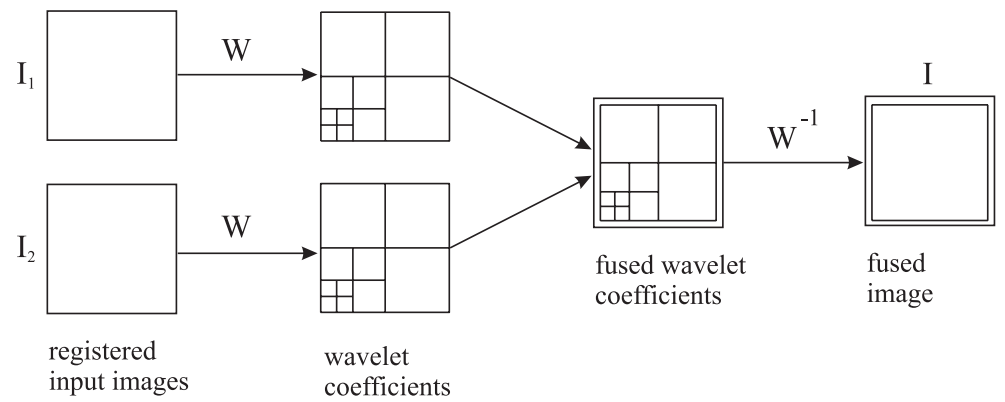

Figure 1: Fusion of the wavelet transforms of two images.

\section{Discrete Wavelet Transform (DWT) Fusion}

The basic idea of all multiresolution fusion schemes is motivated by the human visual system being primarily sensitive to local contrast changes, e.g. the edges or corners. In the case of wavelet transform fusion all respective wavelet coefficients from the input images are combined using the fusion rule $\phi$. Since wavelet coefficients having large absolute values contain the information about the salient features of the images such as edges and lines, a good fusion rule is to take the maximum of the [absolute values of the] corresponding wavelet coefficients. A more advanced area based selection rule is proposed in [1]. The maximum absolute value within a window is used as an activity measure of the central pixel of the window. A binary decision map of the same size as the

\footnotetext{
1 taken from [8]
} 
DWT is constructed to record the selection results based on a maximum selection rule. A similar method was suggested by Burt and Kolczynski [5]. Rather than using a binary decision, the resulting coefficients are given by a weighted average based on the local activity levels in each of the images' subbands. Another method called contrast sensitivity fusion is given in [9]. This method uses a weighted energy in the human perceptual domain, where the perceptual domain is based upon the frequency response, i.e. contrast sensitivity, of the human visual system. This wavelet transform image fusion scheme is an extension to the pyramid based scheme described by the same authors. Finally, a recent publication by Zhang and Blum [10] provides a detailed classification and comparison of multiscale image fusion schemes.

\subsubsection{Implemented Fusion Rules}

Three previously developed fusion rule schemes were implemented using discrete wavelet transform based image fusion:

- maximum selection $(M S)$ scheme: This simple scheme just picks the coefficient in each subband with the largest magnitude;

- weighted average (WA) scheme: This scheme developed by Burt and Kolczynski [5] uses a normalised correlation between the two images' subbands over a small local area. The resultant coefficient for reconstruction is calculated from this measure via a weighted average of the two images' coefficients;

- window based verification (WBV) scheme: This scheme developed by Li et al. [1] creates a binary decision map to choose between each pair of coefficients using a majority filter.

\section{Complex Wavelet Image Fusion}

Figure 2 demonstrates the fusion of two images using the complex wavelet transform. The areas of the images more in focus give rise to larger magnitude coefficients within that region. A simple choose maximum scheme is used to produce the combined coefficient map. The resulting fused image is then produced by transforming the combined coefficient map using the inverse complex wavelet transform. The wavelet coefficient images show the orientated nature of the complex wavelet subbands. Each of the clocks' hands which are pointing in different directions are picked out by the differently orientated subbands. 
All of the coefficient fusion rules implemented with the discrete wavelet transform can also be implemented with the complex wavelet transform. In this case, however, they must be applied to the magnitude of the DT-CWT coefficients as they are complex.

Although extremely computationally efficient, the discrete wavelet transform is not shift invariant. Shift invariance within wavelet transform image fusion is essential for the effective comparison of coefficient magnitudes used by the fusion rule $\phi$. This is because the magnitude of a coefficient within a shift variant transform will often not reflect the true transform content at that point. The shift variance within a DWT is a result of the subsampling necessary for critical decimation. The shift invariant discrete wavelet transform (SIDWT) was an initial attempt to integrate shift invariance into a DWT by discarding all subsampling. The SIDWT is therefore considerably overcomplete. It has been used for image fusion as reported in [7] with much improved results over the standard DWT techniques. The motivation for using the DT-CWT for image fusion is its shift invariance together with its reduced overcompleteness when compared with the SIDWT. The improved directional selectivity of the DT-CWT should also provide improved results over the SIDWT.

The complex wavelet transform was used with the filters given in [3] designed for improved shift invariance.

\section{Experimental Fusion Method Comparison}

Meaningful comparison of image fusion methods is often dependant on the application. For some applications (e.g. medical image fusion) the aim of the fusion is to combine perceptually salient image elements such as edges and high contrast regions. Evaluation of fusion techniques for such applications can only be effectively based on a perceptual comparison. For other applications (such as multifocus image combination) computational measures can also be used for fusion evaluation. We therefore split the comparison into two types: qualitative comparisons and quantitative comparisons.

\subsection{Qualitative Comparisons}

Two image pairs fused using four fusion methods are displayed in figures 3 and 4 for visual comparison. Close inspection of the images in figure 4 shows that the DT-CWT is able to preserve the subtle texture regions of the brain in the MR image better than any of the other methods. This ability is better retained when using the simple MS coefficient fusion rule. The other fusion rules have the effect of blurring such textures. Ringing effects 

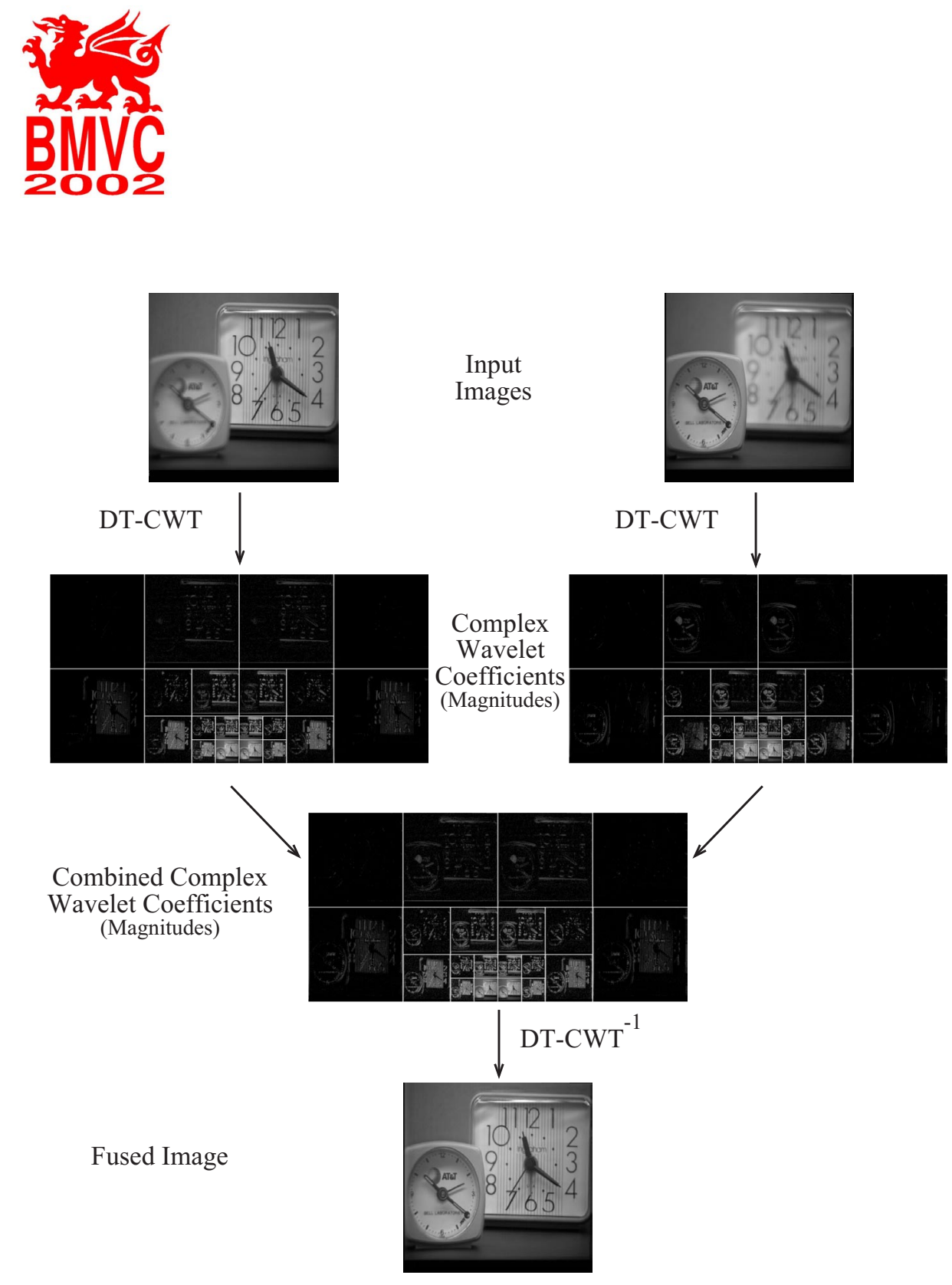

Figure 2: The image fusion process using the DT-CWT and two registered multifocus clock images

are very evident within all the DWT fused results. This is true but less noticeable with the DT-CWT fused images. The multiscale edge fusion results preserve the important edge information from the input images.

\subsubsection{DWT versus DT-CWT}

Figures 3(a) and 3(b) show a pair of multifocus test images that were fused for a closer comparison of the DWT and DT-CWT methods. Figures 3(d) and 3(e) show the results of a simple MS method using the DWT and DT-CWT, respectively. These results are clearly superior to the simple pixel averaging result shown in 3(c). They both retain a perceptually 
acceptable combination of the two "in focus" areas from each input image. An edge fusion result is also shown for comparison (figure 3(f)) [8]. Upon closer inspection however, there are residual ringing artefacts found in the DWT fused image not found within the DT-CWT fused image. Using more sophisticated coefficient fusion rules (such as WBV or WA) the DWT and DT-CWT results were much more difficult to distinguish. However, the above comparison when using a simple MS method reflects the ability of the DT-CWT to retain edge details without ringing.
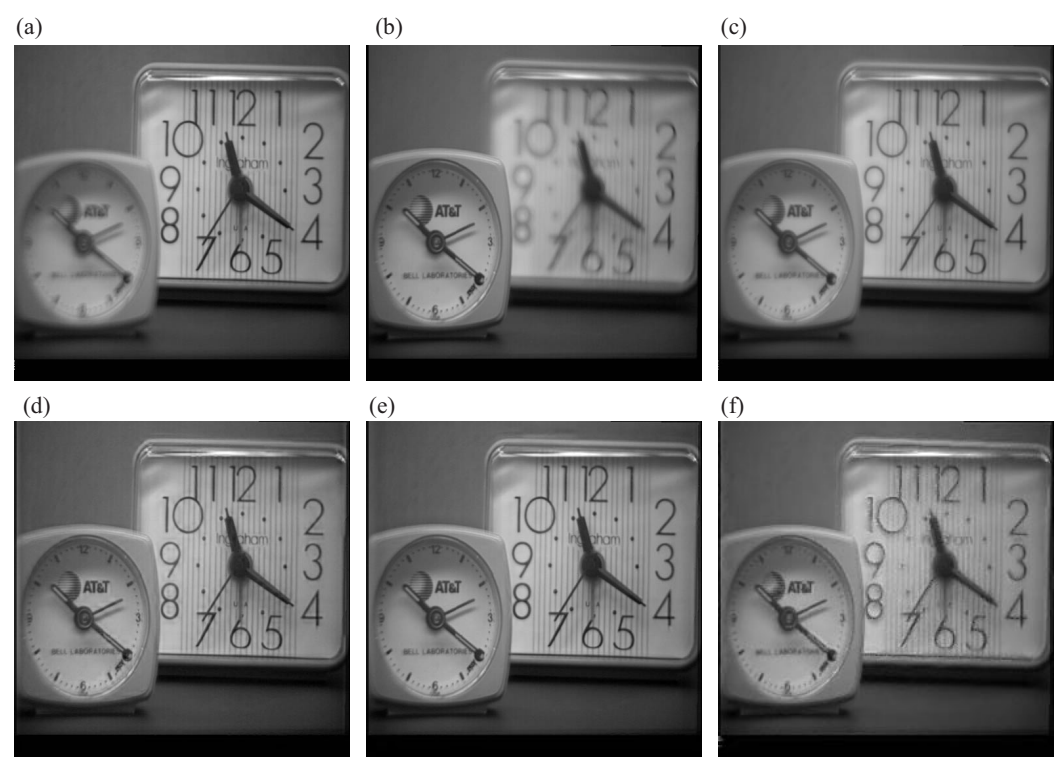

Figure 3: (a) First image of the multifocus test set. (b) Second image of the multifocus test set. (c) Fused image using average pixel values. (d) Fused image using DWT with an MS fuse rule. (e) Fused image using DT-CWT with an MS fuse rule. (f) Fused image using multiscale edge fusion (point representations).

\subsection{Quantitative Comparisons}

Often the perceptual quality of the resulting fused image is of prime importance. In these circumstances comparisons of quantitative quality can often be misleading or meaningless. However, a few authors $[1,7,10]$ have attempted to generate such measures for applications where their meaning is clearer. Figures 3(a) and 3(b) reflect such an application: fusion of two images of differing focus to produce an image of maximum focus. Firstly, a "ground truth" image needs to be created that can be quantitatively compared to the fusion result images. This is produced using a simple cut-and-paste technique, physi- 

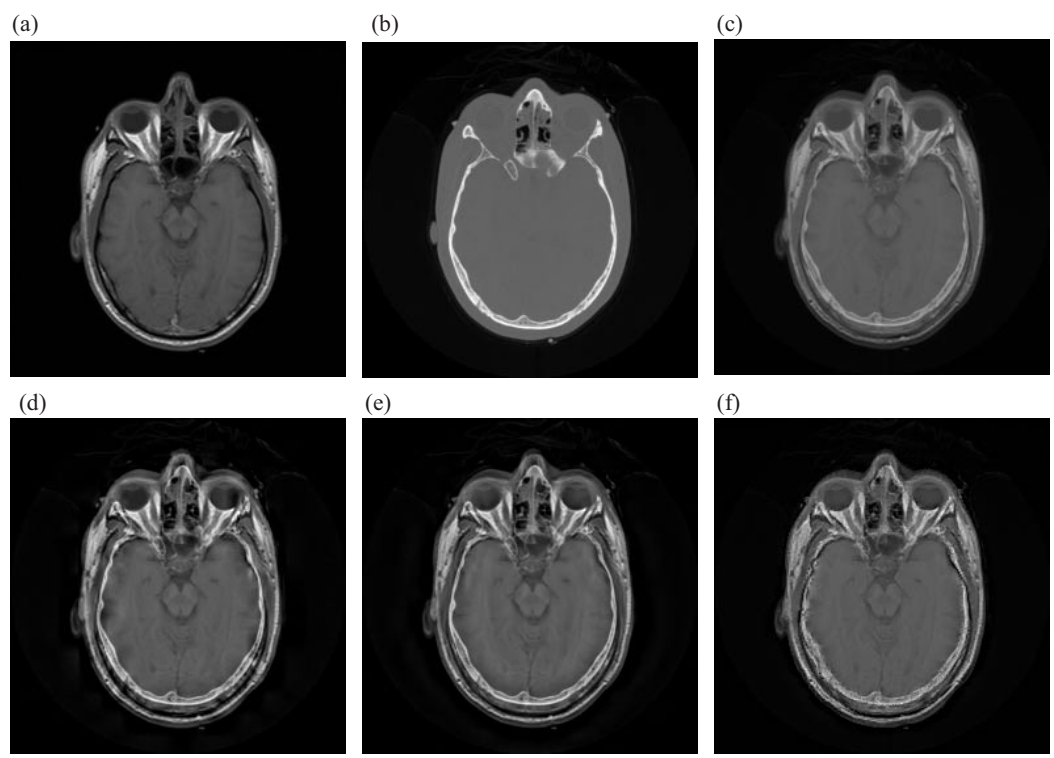

Figure 4: (a) First image (MR) of the medical test set. (b) Second image (CT) of the medical test set. (c) Fused image using average pixel values. (d) Fused image using DWT with an MS fuse rule. (e) Fused image using DT-CWT with an MS fuse rule. (f) Fused image using multiscale edge fusion (point representations).

cally taking the "in focus" areas from each image and combining them. The quantitative measure used to compare the cut-and-paste image to each fused image was taken from [1]

$$
\rho=\sqrt{\frac{\sum_{i=1}^{N} \sum_{j=1}^{N}\left[I_{g t}(i, j)-I_{f d}(i, j)\right]^{2}}{N^{2}}},
$$

where $I_{g t}$ is the cut-and-paste "ground truth" image, $I_{f d}$ is the fused image and $N$ is the size of the image. Lower values of $\rho$ indicate greater similarity between the images $I_{g t}$ and $I_{f d}$ and therefore more successful fusion in terms of quantitatively measurable similarity.

Table 1 shows the results for the various methods used. The average pixel value method gives a baseline result. The PCA method gave an equivalent but a slightly worse result. These methods have poor results relatively to the others. This was expected as they have no scale selectivity. Results were obtained for the DWT methods using all the biorthogonal wavelets available within the Matlab (5.0) Wavelet Toolbox. Similarly, results were obtained for the DT-CWT methods using all the shift invariant wavelet filters described in [3]. Results were also calculated for the SIDWT using the Haar wavelet and the bior2.2 Daubechies wavelet. The table 1 shows the best results for all filters for each 
method. For all filters, the DWT results were worse than their DT-CWT equivalents. Similarly, all the DWT results were worse than their SIDWT equivalents. This demonstrates the importance of shift invariance in wavelet transform fusion. The DT-CWT results were also better than the equivalent results using the SIDWT. This indicates the improvement gained from the added directional selectivity of the DT-CWT over the SIDWT. The WBV and WA methods performed better than MS with equivalent transforms as expected, with WBV performing best for both cases. All of the wavelet transform results were decomposed to four levels. In addition, the residual low pass images were fused using simple averaging and the window for the WA and WBV methods were all set to $3 \times 3$.

\begin{tabular}{||c|c||}
\hline Fusion Method & $\rho$ \\
\hline Average pixel fusion & 8.3553 \\
PCA (MS fusion rule) & 8.3737 \\
DWT (MS fusion rule) & 8.2964 \\
SIDWT (MS fusion rule) & 7.2080 \\
DT-CWT (MS fusion rule) & 7.1184 \\
DDWT - all wavelet coefficients (MS fusion rule) & 7.6165 \\
DDWT - point representations only (MS fusion rule) & 8.4517 \\
DWT (WA fusion rule) & 7.6551 \\
DT-CWT (WA fusion rule) & 7.1043 \\
DWT (WBV fusion rule) & 7.5271 \\
DT-CWT (WBV fusion rule) & 6.9540 \\
\hline
\end{tabular}

Table 1: Quantitative results for various fusion methods.

\subsection{Effect of Wavelet Filter Choice for DWT and DT-CWT Based Fusion}

There are many different choices of filters to effect the DWT transform. In order not to introduce phase distortions, using filters having a linear phase response is a sensible choice. To retain a perfect reconstruction property, this necessitates the use of biorthogonal filters. MS fusion results were compared for all the images in figures 3 and 4 using all the biorthogonal filters included in the Matlab (5.0) Wavelet Toolbox. Likewise there are also many different choices of filters to effect the DT-CWT transform. MS fusion results 
were compared for all the same three image pairs using all the specially designed filters given in [3].

Qualitatively all the DWT results gave more ringing artefacts than the equivalent DTCWT results. Different choices of DWT filters gave ringing artefacts at different image locations and scales. The choice of filters for the DT-CWT did not seem to alter or move the ringing artefacts found within the fused images. The perceived higher quality of the DT-CWT fusion results compared to the DWT fusion results was also reflected by a quantitative comparison.

\section{Summary}

The aim of this paper has been to compare image fusion using the DT-CWT with existing fusion techniques. For an effective fusion of images a technique should aim to retain important features from all input images. These features often appear at different positions and scales. Multiresolution analysis tools such as the wavelet transform are therefore ideally suited to image fusion. Simple non-multiresolution methods for image fusion (such as averaging and PCA methods) have produced limited results (see table 1).

\subsection{DWT Fusion}

The DWT fusion methods provide computationally efficient image fusion techniques. Various fusion rules for the selection and combination of subband coefficients increase the quality (perceptual and quantitatively measurable) of image fusion in specific applications.

\subsection{DT-CWT Fusion}

The developed DT-CWT fusion techniques provide better quantitative and qualitative results than the DWT at the expense of increased computation. The DT-CWT method is able to retain edge information without significant ringing artefacts. It is also good at faithfully retaining textures from the input images. All of these features can be attributed to the increased shift invariance and orientation selectivity of the DT-CWT when compared to the DWT. A previously developed shift invariant wavelet transform (the SIDWT) has been used for image fusion [7]. However, the SIDWT suffers from excessive redundancy. The SIDWT also lacks the directional selectivity of the DT-CWT. This is reflected in the superior quantitative results of the DT-CWT (see table 1). Various fusion rules for the 
election and combination of subband coefficients increases the quality (perceptual and quantitatively measurable) of image fusion in specific applications. The DT-CWT has the further advantage that the phase information is available for analysis. However, after an initial set of experiments using the notion of phase coherence, no improvement in fusion performance has been achieved.

\section{References}

[1] H. Li, B.S. Manjunath, and S.K. Mitra. Multisensor image fusion using the wavelet transform. Graphical Models and Image Processing, 57:235-245, 1995.

[2] J.L. Moigne and R.F. Cromp. The use of wavelets for remote sensing image registration and fusion. Technical Report TR-96-171, NASA, 1996.

[3] N.G. Kingsbury. The dual-tree complex wavelet transform with improved orthogonality and symmetry properties. IEEE International Conference on Image Processing, pages 375-378, September 2000.

[4] L.J. Chipman, T.M. Orr, and L.N. Lewis. Wavelets and image fusion. IEEE Transactions on Image Processing, 3:248-251, 1995.

[5] P.J. Burt and R.J. Kolczynski. Enhanced image capture through fusion. Proceedingsof the 4th International Conference on Computer Vision, pages 173-182, 1993.

[6] O. Rockinger. Pixel-level fusion of image sequences using wavelet frames. In Mardia, K. V., Gill, C. A., and Dryden, I. L., editor, Proceedings in Image Fusion and Shape Variability Techniques, pages 149-154., Leeds, UK, 1996.

[7] O. Rockinger. Image sequence fusion using a shift invariant wavelet transform. IEEE Transactions on Image Processing, 3:288-291, 1997.

[8] S. Nikolov, P.R. Hill, D.R. Bull, C.N. Canagarajah. Wavelets for image fusion. In A. Petrosian and F. Meyer, editors, Wavelets in Signal and Image Analysis, from Theory to Practice. Kluwer Academic Publishers, 2001.

[9] T.A. Wilson, S.K. Rogers, and L.R. Myers. Perceptual based hyperspectral image fusion using multiresolution analysis. Otical Engineering, 34(11):3154-3164, 1995.

[10] Z. Zhang and R. Blum. A categorization of multiscale-decomposition-based image fusion schemes with a performance study for a digital camera application. Proceedings of the IEEE, pages 1315-1328, August 1999. 